\title{
Über die Sinne in der Medizin
}

\section{Eberhard Wolff}

PD Dr. rer. soc., Mitglied der Redaktion Medizingeschichte

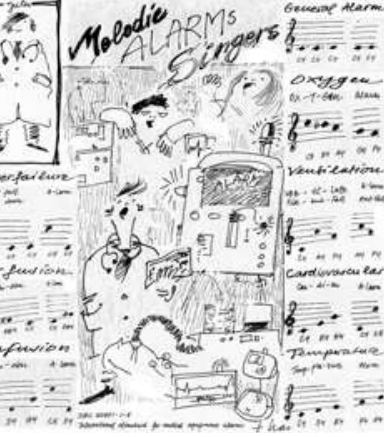

1 Reiser SJ. Technology and the use of the senses in twentieth century medicine. In: Bynum WF, Porter R (Hrsg.). Medicine and the five senses. Cambridge: Cambridge University Press; 1993. S. 262-73. 2 Stolberg M. Die Harnschau. Eine Kultur- und Alltagsgeschichte. Köln: Böhlau; 2009.
Tönt vielleicht noch der wild-lustige Cartoon «Melodie Alarms Singers» aus Heft 9 dieses Blattes in Ihrem Hinterkopf (s. links)? Darin hat Jules all die unterschiedlichen Warntöne in Noten gefasst, die medizinische Überwachungsgeräte von sich geben. Da frage ich mich doch stutzig: Ist das moderne technisierte Spital denn nicht kalt und "unsinnlich» - oder zumindest hoffnungslos in den Sehsinn eingezwängt? Früher, wurde da in der Medizin nicht viel mehr gehört oder abgehorcht, gerochen oder gefühlt?

Vor gut zwanzig Jahren schrieb der MedizintechnikForscher Stanley J. Reiser [1], dass die vielen neuen Diagnosegeräte seit etwa 1850 - vom Thermometer über das Röntgen bis zum «Labor» - die sensuelle körperliche Untersuchung der Patienten ersetzt hätten. Die instrumentelle Diagnose lieferte nun «objektivere» Daten und war einfacher zu kommunizieren. Die sinnenvolle körperliche Untersuchung der Patienten sei dabei in Vergessenheit geraten. Den Ärzten könnte deshalb die unmittelbare Verbindung zu den Patienten und ihrer Persönlichkeit abhanden kommen.

Zugegeben: Mit ein paar Klicks oder Häkchen ein Blutbild in Auftrag zu geben und im Gegenzug Zahlenreihen $\mathrm{zu}$ erhalten ist nicht so sinnenfroh wie das frühere Fühlen und Beschreiben des Pulses. Oder ein deftigeres Beispiel: die Harnschau. Sie war immerhin von der Antike bis zum Anbruch der Moderne [2] ein diagnostisches Standardverfahren der Ärzte und Heiler, das gerade auch von der Patientenschaft gerne nachgefragt wurde. Der erfolgreiche Wundarzt Michel Schüppach aus dem Emmental (1707-1781) etwa wurde nicht zuletzt wegen seiner uroskopischen Künste aufgesucht. Auch Goethe zählte zu den Besuchern.

Die Harnschau ist in der Tat das Paradebeispiel einer multisensualen Diagnostik, das zeigen uns die damaligen Lehrbücher: Der Blick auf die Farbe des Urins lieferte erste Hinweise auf die Krankheit der Patienten; mit der Nase wurde der Geruch des Urins bestimmt und auf der Zunge sein Geschmack erkundet; das Gefühl, das der Urin auf der Haut auslöste, lieferte taktile Anhaltspunkte; das Geräusch, das der Urin beim Wasserlassen oder Ausgiessen machte, die akustischen. Das ist wirklich grosses Kino aller Sinne! In der historischen Praxis schrumpfte die prall sinnenfrohe Diagnose jedoch schnell wieder auf eine
Harn-Schau zusammen. Alles andere waren «Nebenkriterien» der Blickdiagnose. Stand die Harnschau an sich schon immer etwas im Ruch des Betrügerischen, so galt dies insbesondere für das Harnriechen und Harnschmecken. «Die Adlernase in das Harnglas zu stecken", so eine Würzburger medizinische Dissertation von 1601, war so sehr mit Vorstellungen des Ekels besetzt, dass diese Praxis nach Ansicht verschiedener damaliger Autoren eine Gefahr für den Status und die Würde der Ärzte darstellte.

Im Alltag vor allem der akademischen Ärzte war die Harnschau meist wirklich nur eine Schau, keine Harnriecherei oder -schmeckerei. Auf zahlreichen frühneuzeitlichen Ölgemälden wird das Harnglas fast immer hoch, gegen das Licht, gehalten. Die alchimistischen Anhänger des Paracelsus verabschiedeten sich noch weiter vom unmittelbaren Sinneseindruck, indem sie den Urin zum Beispiel destillierten und die entstandenen Ablagerungen untersuchten. Das «unsinnliche» moderne Labor lässt grüssen.

Gerade umgekehrt verhält es sich meines Erachtens mit den modernen Medizingeräten. Man sagt, sie seien sinnenfeindlich. Aber, um an Jules' Cartoon anzuknüpfen: Sie piepsen nicht nur - oder knattern, dass man einen Gehörschutz braucht. Das Ultraschallgerät streicht mit glibberiger Gleitmasse sanft über die Haut. Geräte haben Sinnesorgane, von denen wir nur träumen können. Der Sonograph kann die Impedanz von Materialien wahrnehmen. Können Sie das? Ein MRI kann die elektrische Spannung wahrnehmen, die ein Atomkern absondert, nachdem er in ein hochfrequentes Magnetfeld geraten ist (oder so ähnlich). Ich mit meinen fünf Sinnen bin in dieser Hinsicht taubblind. Damit wir sinnenbeschränkte Menschen daran teilhaben können, übersetzen MRIs ihre Wahrnehmungen transsensuell in immer sinnlichere optische Repräsentationen [1]. Dankeschön!

Geben wir damit unsere eigene sinnliche Wahrnehmung auf und an die Maschinen ab? Ich möchte den Arzt oder die Ärztin sehen, die das akute Abdomen nicht auch vorsichtig abtastet, die Eltern, die neben dem Fiebermessen des Kindes nicht auch den Handrücken auf die Stirn legen. Ich halte heutigen Kulturpessimisten entgegen, dass die moderne Medizin, auch die hochtechnisierte, überaus sinnlich ist. Trotz allem. Man muss das nur wahrnehmen - mit allen Sinnen. 